\title{
artigo
}

Teixeira, J.G.; Vieira, B.C.; França, S.D.M.; Alvino, C.C.M.; Godoi, B.O.; Bontempo, A.P.S.;

Gestantes COVID-19 positivo, trabalho de parto e risco de transmissão vertical: revisão sistemática

\section{Gestantes COVID-19 positivo, trabalho de parto e risco de transmissão vertical: revisão sistemática}

\author{
COVID-19 positive pregnant women, childbirth and risk of vertical transmission: systematic review \\ COVID-19 mujeres embarazadas positivas, parto y riesgo de transmisión vertical: revisión sistemática
}

\begin{abstract}
RESUMO
Gestantes são afetadas desproporcionalmente por doenças respiratórias. Estas estão relacionadas a altas taxas de mortalidade. Objetivos: Observar os manejos com gestantes COVID-19 positivo, o risco da transmissão vertical e os cuidados durante o trabalho de parto. Métodos: Revisão sistemática realizada em junho de 2020, na Biblioteca Virtual em Saúde; descritores "GESTANTE" e "COVID-19", assunto principal "infecção por Coronavírus". Resultados: Foram encontrados 130 artigos, após critérios de elegibilidade, foram selecionados 18 . Gestantes infectadas no $3^{\circ}$ trimestre apresentaram sintomas semelhantes aos demais pacientes. Parto cesáreo foi o mais realizado, não houve evidências de transmissão vertical nem pelo leite materno. Orientam-se medidas de segurança, como isolamento e uso de equipamentos de proteção individual durante amamentação. Conclusão: Os manejos com gestantes infectadas são semelhantes aos de outros pacientes, o parto cesáreo foi o mais utilizado e não é essencial. Não houve evidências de transmissão vertical ou contaminação pelo leite materno.
\end{abstract}

DESCRITORES: COVID-19; Gestante; Transmissão Vertical.

\section{ABSTRACT}

Pregnant women are disproportionately affected by respiratory diseases. These are related to high mortality rates. Objectives: To observe the management with positive COVID-19 pregnant women, the risk of vertical transmission and care during labor. Methods: Systematic review carried out in June 2020, at the Virtual Health Library, descriptors "GESTANTE" and "COVID-19", main subject "Coronavirus infection". Results: 130 articles were found, after eligibility criteria were selected 18. Pregnant women infected in the 3rd trimester had symptoms similar to the other patients. Cesarean deliveries were the most performed, there was no evidence of vertical transmission or by breast milk. Safety measures are recommended, such as isolation and use of personal protective equipment during breastfeeding. Conclusion: The management with infected pregnant women is similar to that of other patients, cesarean delivery was the most used and is not essential. There was no evidence of vertical transmission or contamination by breast milk.

DESCRIPTORS: COVID-19; Pregnant; Vertical transmission.

\section{RESUMEN}

Las mujeres embarazadas se ven afectadas de manera desproporcionada por enfermedades respiratorias. Estos están relacionados con altas tasas de mortalidad. Objetivos: Observar el manejo con gestantes COVID-19 positivo, el riesgo de transmisión vertical y el cuidado durante el parto. Métodos: Revisión sistemática realizada en junio de 2020, en la Biblioteca Virtual en Salud, descriptores "GESTANTE" y "COVID-19", tema principal "Infección por coronavirus". Resultados: Se encontraron 130 artículos, luego de seleccionar los criterios de elegibilidad 18. Las mujeres embarazadas infectadas en el 3er trimestre tenían síntomas similares a los de las demás pacientes. Los partos por cesárea fueron los más realizados, no hubo evidencia de transmisión vertical ni por leche materna. Se recomiendan medidas de seguridad, como aislamiento y uso de equipo de protección personal durante la lactancia. Conclusión: El manejo con gestantes infectadas es similar al de otras pacientes, la cesárea fue la más utilizada y no es imprescindible. No hubo evidencia de transmisión vertical o contaminación por la leche materna.

DESCRIPTORES: COVID-19; Embarazada; Transmisión vertical.

RECEBIDO EM: 28/10/2020 APROVADO EM: 10/11/2020

\section{Jackson Gois Teixeira}

Graduando de Enfermagem - Centro Universitário do Distrito Federal - UDF.

ORCID: 0000-0003-2804-1130 


\title{
Brenda Christina Vieira
}

Graduanda de Enfermagem - Centro Universitário do Distrito Federal - UDF.

ORCID: 0000-0001-5759-619X

\section{Silvana Dias de Macedo França}

Graduanda de Enfermagem - Centro Universitário do Distrito Federal - UDF.

ORCID: 0000-0001-9412-1409

\section{Camylla Cristina de Melo Alvino}

Graduanda de Fisioterapia - Centro Universitário do Distrito Federal - UDF.

ORCID: 0000-0002-6965-0875

\section{Bruna de Oliveira Godoi}

Graduação em Nutrição pela Universidade Paulista (2012), especialização em Nutrição Clínica

Funcional e Fitoterápica pela Faculdade Laboro (2016). Graduanda em Fisioterapia pelo

Centro Universitário do Distrito Federal - UDF.

ORCID: 0000-0002-5026-5643

\begin{abstract}
Albênica Paulino dos Santos Bontempo
Graduada em Fisioterapia pela Universidade Paulista - UNIP (2008), mestre em Ciências e Tecnologias em Saúde pela Universidade de Brasília - UNB, especialista em Fisioterapia Aplicada da Saúde da Mulher, Docência do ensino superior e profissionalizante e em Anatomia Humana e Patologias Associadas. Membro da Associação Brasileira de Fisioterapia em Saúde da Mulher Humana e Patologias Associadas. Membro da Associação Brasileira de Fisioterapia em Saúde da Mulher - ABRAFISM. Docente no Centro Universitário do Distrito Federal - UDF e no curso de especialização em Fisioterapia Aplicada a Saúde da Mulher pelo Instituto Inspirar. Professora Orientadora das Ligas Acadêmicas de Gerontologia e de Cardiologia do UDF. Atua como fisioterapeuta com experiência na área de saúde da mulher e gerontologia.

ORCID: 0000-0001-6942-1207
\end{abstract}

\section{INTRODUÇÃO}

0 vírus da COVID-19 manifestou-se em dezembro de 2019 em Wuhan na China e espalhou-se mundialmente, provocando uma grave crise na saúde pública, que foi classificada pela Organização Mundial de Saúde (OMS) como pandemia ${ }^{1}$.

A gravidez é um estado de imunossupressão parcial que torna a gestante mais vulnerável a infecções virais². As gestantes são afetadas desproporcionalmente por doenças respiratórias, que estão relacionadas a altas taxas de morte materna, aborto espontâneo, teratogenicidade e restrição de crescimento intrauterino ${ }^{3}$. Neste contexto, embora a maioria dos casos da COVID-19 tenha sintomas leves, ainda é necessário atenção devido a fatos históricos, como os vividos em epidemias severas, a exemplo da síndrome respiratória MERS-COV, em que cerca de $1 / 3$ das gestantes infectadas (GI) vieram a óbito no Oriente Médioº.

A via de parto também é alvo de pes- quisas relacionadas à COVID-19, pois não se sabe ao certo quais cuidados a equipe de assistência à parturiente precisa ter ${ }^{3}$. Embora se saiba que infecção viral materna pode afetar o feto no primeiro trimestre da gestação, ainda são poucos os estudos relacionados à doença, sendo importante também analisar a possibilidade de transmissão vertical ${ }^{4}$.

Diante desse cenário, este estudo almeja realizar uma revisão sistemática para observar os manejos com gestantes $\mathrm{CO}$ VID-19 positivo, o risco da transmissão vertical e os cuidados durante o parto.

\section{MÉTODO}

Revisão sistemática, realizada na Biblioteca Virtual em Saúde em junho/2020. Esta base foi escolhida por conter fontes de informação nacionais e internacionais disponíveis na integra e de forma gratuita (BIREME, MEDLINE, LILACS, Biblioteca Cochrane, LIS, DirEve, Leyes e SeCS). Foram seguidas as orientações do "Relatório Preferidos para
Revisões Sistemáticas e Meta-Análises" (PRISMA). Foram utilizados os descritores "GESTANTE" e "COVID-19" e os filtros: texto disponível na íntegra, assunto principal "infecção por Coronavírus", limite gravidez, publicado em 2020. Realizaram a busca 3 pesquisadores independentemente.

A seleção seguiu os critérios de elegibilidade, priorizando artigos relacionados a "gestantes, parturientes e recém-nascidos (RNs)". Foram excluídos estudos sobre medicamentos, vacinas, exames, artigos de opinião e cartas editoriais. Todo o processo teve 3 etapas: leitura dos títulos, resumos e integral. Foi realizado simultaneamente pelos pesquisadores, que compararam suas seleçôes e discutiram as divergências. Por fim, foram extraídos os resultados e conclusões mais relevantes.

\section{RESULTADOS}

Foram localizados 130 artigos; em seguida, removidos os duplicados e indisponíveis. Restaram 18 após o uso dos filtros e 


\section{artigo}

Teixeira, J.G.; Vieira, B.C.; França, S.D.M.; Alvino, C.C.M.; Godoi, B.O.; Bontempo, A.P.S.;

Gestantes COVID-19 positivo, trabalho de parto e risco de transmissão vertical: revisão sistemática

Figura 1. Fluxograma descrevendo a estratégia de busca e seleção de estudos dos itens Preferenciais de Relatórios para Revisões Sistemáticas e Meta-Análises (PRISMA).

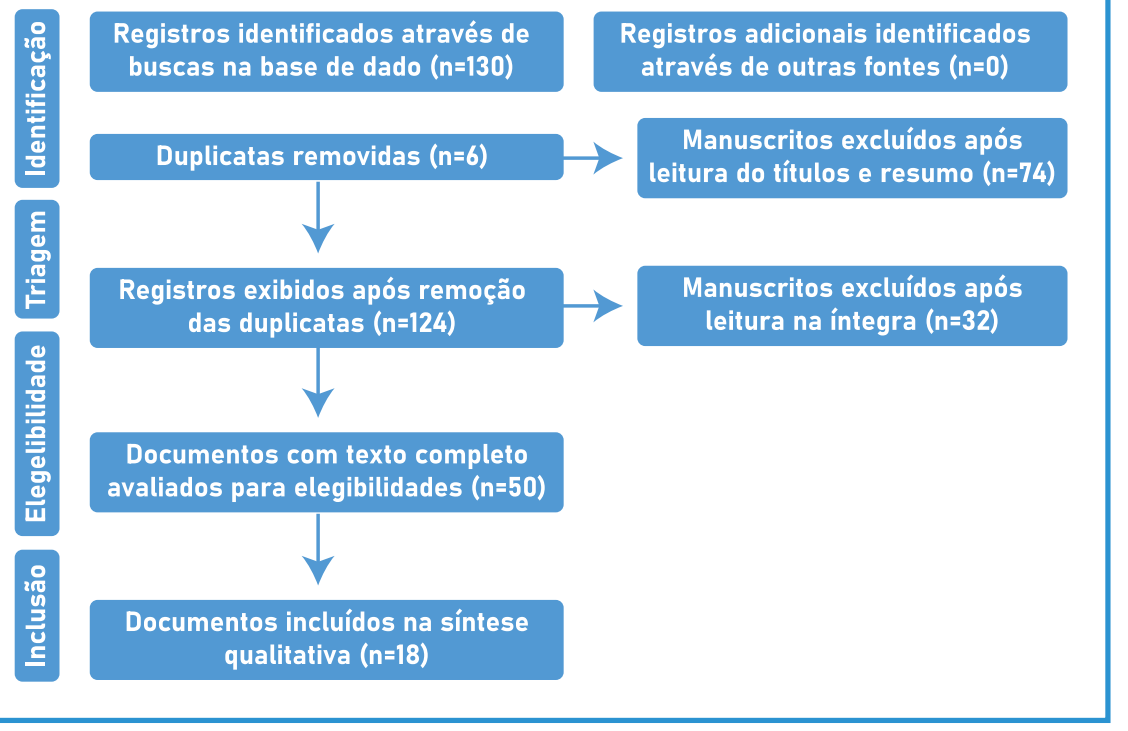

aplicação dos critérios de elegibilidade. Os detalhes da busca estão esquematizados no fluxograma da figura 1 .

O Quadro 1 apresenta a síntese dos principais achados de cada artigo.

\section{DISCUSSÃO}

A gravidez é como um estado de imunossupressão parcial que torna a gestante mais vulnerável a infecções virais. Isso ocorre devido a mudanças fisiológicas gestacionais que podem facilitar a rápida progressão de doenças respiratórias ${ }^{1,2}$. Apesar disso, gestantes têm risco relativamente baixo para COVID-19, pois são jovens, saudáveis e geralmente assintomáticas ${ }^{5,6}$. Muitos autores observaram que GI apresentaram sintomas semelhantes aos demais pacientes, mas Smith et al. (2020) relataram que elas apresentam menos sintomas

Quadro 1- Síntese dos principais achados relacionados aos manejos com gestantes COVID-19 positivo, transmissão vertical e os cuidados durante o parto. Brasília-Brasil, 2020.

\begin{tabular}{|c|c|c|}
\hline $\mathbf{N}$ & AUTORES (ANO) & PRINCIPAIS ACHADOS \\
\hline 1 & Baergen, et al. (2020) & EC com 20 placentas de Gl, 10 apresentaram evidência de má perfusão vascular ou trombose vascular fetal. \\
\hline 2 & Carvalho et al. (2020) & $\begin{array}{l}\text { EOR de prontuários de GI e RN. As mães tiveram dificuldade respiratória e precisaram de suporte } \\
\text { ventilatório, coagulação intravascular disseminada, disfunção de múltiplos órgãos e choque. RNs } \\
\text { testaram negativo. }\end{array}$ \\
\hline 3 & Chen, et al. (2020) & $\begin{array}{l}\text { E. Clínico com } 3 \text { placentas de Gl no } 3^{\circ} \text { trimestre com emergência cesariana. Características clínicas se- } \\
\text { melhantes às de pacientes não grávidas, não houve alteração placentária. RNs testaram negativo. }\end{array}$ \\
\hline 4 & Dashraath, et al. (2020) & $\begin{array}{l}\text { RS. Relatos de } 55 \mathrm{Gl} \text { e } 46 \text { RNs, não houve transmissão vertical. Mudanças fisiológicas e mecânicas na } \\
\text { gestação aumentam a suscetibilidade a infecções, particularmente quando o sistema cardiorrespirató- } \\
\text { rio foi afetado, o que levou à rápida progressão para insuficiência respiratória. }\end{array}$ \\
\hline 5 & Herraiz et al. (2020) & $\begin{array}{l}\text { EOR de } 203 \text { partos analisados, } 7 \text { gestantes referiram sintomas, mas apenas } 1 \text { teve RT-PCR positivo. } \\
\text { Das } 194 \text { assintomáticas, } 1 \text { foi positivo. Sintomas semelhantes aos demais pacientes. }\end{array}$ \\
\hline 6 & Indraccolo, U. (2020) & $\begin{array}{l}\text { EC de uma GI. Houve dificuldade para rastrear o caso. Gestantes precisam ser orientadas a relatar rapi- } \\
\text { damente os sintomas, ainda que leves. }\end{array}$ \\
\hline 7 & lqbal, et al. (2020) & $\begin{array}{l}\text { EC de um parto de Gl. Ela e a equipe de assistência usaram EPIs. Não houve o clampeamento tardio } \\
\text { do cordão umbilical nem o contato cutâneo entre mãe e filho. RN testou negativo, foi alimentado com } \\
\text { fórmula e leite materno ordenhado. Mantido longe da mãe até sua recuperação e alta. }\end{array}$ \\
\hline 8 & Lang, G.; Zhao. (2020) & $\begin{array}{l}\text { EC de Gl. Não houve transmissão do vírus no leite materno. Sugere-se isolar a mãe. A amamentação } \\
\text { pode ser praticada após fim do isolamento desde que ela tenha testado negativo. }\end{array}$ \\
\hline 9 & Liu et al. (2020) & $\begin{array}{l}\text { EOR com } 19 \mathrm{Gl} \text {. Partos realizados em sala isolada. RNs foram separados imediatamente de suas mães. } \\
\text { Não houve sofrimento fetal. RNs testaram negativo. }\end{array}$ \\
\hline 10 & Lyra et al. (2020) & $\begin{array}{l}\text { EC de cesariana em Gl, sem complicações. RN foi separado da mãe imediatamente e testou negativo. } \\
\text { A via de parto deve ser escolhida pelas condições obstétricas com condições de trabalho seguras para } \\
\text { equipe de assistência. }\end{array}$ \\
\hline 11 & Mimouni, et al. (2020) & $\begin{array}{l}\text { RS. Não houve evidências de transmissão vertical nem de que a gravidez é um fator de risco para maior } \\
\text { gravidade da doença. Não há evidências da gravidade da doença em RNs assim como da presença do } \\
\text { vírus no leite materno. }\end{array}$ \\
\hline
\end{tabular}




\begin{tabular}{|c|c|c|}
\hline 12 & Peng et al. (2020) & $\begin{array}{l}\text { EC de cesariana em GI com características clínicas semelhantes a outros pacientes. RN teve sofrimento } \\
\text { intrauterino e testou negativo. Recomenda-se cautela sobre a transmissão vertical. }\end{array}$ \\
\hline 13 & Rasmussen, et al. (2020) & $\begin{array}{l}\text { RS. Os dados disponíveis sobre GI são limitados. Deve-se basear nas recomendações de outras infec- } \\
\text { ções respiratórias, como SARS e MERS, que sugerem que grávidas podem ter um curso clínico grave. As } \\
\text { intervenções padrão para controlar qualquer infecção respiratória grave são a base do cuidado para Gl. }\end{array}$ \\
\hline 14 & Shanes et al. (2020) & $\begin{array}{l}\text { Ensaio Clínico com } 16 \text { placentas de Gl, } 1 \text { do } 2^{\circ} \text { trimestre após aborto - apresentou edema de vilosida- } \\
\text { des e hematoma. } 3 \text { do } 30 \text { trimestre tinham características de má perfusão vascular materna e arte- } \\
\text { riopatia. } 1 \text { era hipertensa, apesar da associação com distúrbios hipertensivos e pré-eclâmpsia. Essas } \\
\text { alterações podem refletir um estado inflamatório sistêmico ou hipercoagulável, que influenciam a fi- } \\
\text { siologia placentária. }\end{array}$ \\
\hline 15 & Smith et al. (2020) & $\begin{array}{l}\text { RS. Gl apresentam menos sintomas que a população geral e podem ter RT-PCR* negativo, apesar de } \\
\text { terem sinais de pneumonia viral. A incidência de partos prematuros, baixo peso ao nascer, cesárea e } \\
\text { internação em UTIN foi maior que na população geral. }\end{array}$ \\
\hline 16 & Yang et al. (2020) & $\begin{array}{l}\text { EOR com } 7 \text { RNs ( } 4 \text { prematuros). A infecção em gestantes tardias não causa resultados adversos nos } \\
\text { RNs. Sugere-se isolamento imediato. }\end{array}$ \\
\hline 17 & Yu et al. (2020) & $\begin{array}{l}\text { EOR com } 7 \mathrm{Gl} \text { que apresentaram características clínicas semelhantes a outros pacientes. Os resultados } \\
\text { maternos, fetais e neonatais foram bons. }\end{array}$ \\
\hline 18 & Zhang, et al. (2020) & $\begin{array}{l}\text { EOR comparativo entre gestantes, sendo } 16 \text { infectadas e } 45 \text { não infectadas. Todos partos cesáreos, no } \\
\text { 3o trimestre. Não houve diferença entre os grupos. RNs de Gl testaram negativo. }\end{array}$ \\
\hline
\end{tabular}

e podem ter RT-PCR negativo, apesar de terem sinais de pneumonia viral. ${ }^{7,89}$.

Sobre a via de parto, Herraiz (2020) relatou 1 caso de GI que teve parto eutócico, a termo, sem complicações. Liu et al. (2020) contam que, entre $19 \mathrm{GI}$, somente 1 teve parto normal, e Lyra et al. (2020) relataram o caso de uma GI que fez cesariana porque a equipe de assistência considerou o risco de contaminação do RN5,10,11. Yang et al. (2020) e Zang et al. (2020) também relataram partos cesáreos preventivos. Já Baergen (2020) e Pu e Liu (2020) relataram casos de parto espontâneo; as mães permaneceram com seus RNs e com os pais na sala de parto, sem complicações ${ }^{15,16}$. Os autores orientam, na escolha da via de parto, a considerar as condições obstétricas da parturiente e as condições de segurança da equipe ${ }^{5,10,11}$. Segundo Chen et al. (2020), a via de parto não necessariamente precisa ser cesárea, mas é recomendada em casos de hipóxia materna, choque séptico e/ou sofrimento fetal ${ }^{4}$. O Ministério da Saúde não recomenda a ocorrência de partos na água, para segurança da equipe e do RN, uma vez que o vírus também é eliminado nas fezes ${ }^{14 .}$

Três estudos analisaram a placenta e não observaram alterações específicas, mas relataram casos de má distribuição de oxi- gênio materno e trombose, referidas como lesão vascular placentária ${ }^{15,17,18}$. Sobre a transmissão vertical, ainda não há evidências robustas ${ }^{19}$. Contudo, a orientação dos pesquisadores é que o cordão umbilical seja clampeado e cortado rapidamente durante o parto para evitar a passagem de sangue

\section{O Ministério}

\section{da Saúde não}

\section{recomenda a}

ocorrência de

partos na água, para segurança da equipe e do RN, uma vez que o vírus também é eliminado nas fezes. periférico materno e líquido amniótico ${ }^{15,16}$. Embora alguns RNs tenham sido internados em UTIN com dificuldades respiratórias e necessidade de ventilação assistida, os sintomas não foram graves e a recuperação foi rápida. Essas ocorrências não tiveram relação com o vírus ${ }^{12,13}$. No entanto, Vivanti et al (2020) relataram o ocorrido no Paris Saclay University Hospitals como o primeiro caso comprovado de transmissão vertical do vírus por via placentária ${ }^{20}$.

Não houve transmissão do vírus pelo leite materno ${ }^{21}$. Contudo, estudos sugerem que mães infectadas sejam isoladas $^{22,23}$. Porém Dashraath et al. (2020) relataram que $\mathrm{RNs}$ foram amamentados normalmente, seguindo protocolos de segurança, e não houve contaminação ${ }^{1}$. Os cuidados essenciais da mãe com o $\mathrm{RN}$ são o uso de EPIs, como aventais, luvas, máscaras cirúrgicas e proteção ocular, para evitar que gotículas com carga viral cheguem ao RN. Em casos de GI, deve-se suspender o contato cutâneo ${ }^{24}$. As diretrizes do Royal College of Obstetricians and Gynecologists afirmam que é possível amamentação e alojamento conjunto desde que sejam seguidas as medidas de segurança. Outrossim, a OMS apoia a amamentação para mães mesmo que infectadas ${ }^{25}$. 


\section{artigo}

Teixeira, J.G.; Vieira, B.C.; França, S.D.M.; Alvino, C.C.M.; Godoi, B.O.; Bontempo, A.P.S.;

Gestantes CoVID-19 positivo, trabalho de parto e risco de transmissão vertical: revisão sistemática

\section{CONCLUSÃO}

Gestantes infectadas, no $3^{\circ}$ trimestre, apresentaram sintomas semelhantes aos demais pacientes. Parto cesáreo foi o mais utilizado. Não houve evidências de trans- missão vertical ou pelo leite materno. Os achados não são conclusivos, recomenda-se a realização de mais estudos.

\section{REFERÊNCIAS}

1. Dashraath P, Wong JLJ, Lim MXK, Lim LM, Li S, Biswas A, et al. Coronavirus disease 2019 (COVID-19) pandemic and pregnancy. AJOG. 2020;222(6):521-531. doi: 10.1016/j.ajog.2020.03.021.

2. Liang $\mathrm{H}$, Acharya G. Novel Coronavirus disease (COVI-19) in pregnacy: What clinical recommendations to follow? AOGS. 2020;99(4):439-442. doi: 10.1111/aogs.13836

3. Hamed MA. An overview on COVID-19: reality and expectation. Bull Natl Res Cent. 2020;44(1):86. doi: 10.1186/s42269-02000341-9.

4. Chen H, Guo J, Wang C, Luo F, Yu X, Zhang W et al. Clinical characteristics and intrauterine vertical transmission potential of COVID-19 infection in nine pregnant women: a retrospective review of medical records. The Lancet. 2020;395:809-815. doi: 10.1016/ S0140-6736(20)30360-3.

5. Herraiz I, Folgueira D, Villalaín C, Forcén L, Delgado R, Galindo A. Universal screenig for SARS-Cov-2 before labor admission during Covid-19 pandemic in Madrid. Journal of Perinatal Medicine. 2020. Doi: 10.1515/jpm-2020-0236.

6. Yu N, Li W, Kang Q, Xiong Z, Wang S, Lin X, et al. Clinical features and obstetric and neonatal outcomes of pregnant patients with COVID-19 in Wuhan, China: a retrospective, single-centre, descriptive study. The Lancet. 2020;20(5):559-564. doi: 10.1016/S14733099(20)30176-6.

7. Indraccolo U. A pregnant woman and the SARS-CoV-2 infection: how are barriers easily crossed? Recenti Prog Med. 2020;111(4):259-260. doi: 10.1701/3347.33190.

8. Iqbal SN, Overcash R, Mokhtari N, Saeed H, Gold S, Auguste T, et al. An Uncomplicated Delivery in a Patient with Covid-19 in the United States. N Engl J Med. 2020;382(16): e34. doi: 10.1056 / NEJMc2007605.

9. Smith V, Seo D, Warty R, Payne O, Salih M, Chin K L, et al. Maternal and neonatal outcomes associated with COVID-19 infection: A systematic review. PO. 2020;15(6): e0234187. doi: 10.1371/journal. pone.0234187.

10. Liu W, Wang J, Li W, Zhou Z, Liu S, Rong Z. Clinical characteristics of 19 neonates born to mothers with COVID-19. FM. 2020;14(2):193-198. doi: 10.1007/s11684-020-0772-y.

11. Lyra, J. et al. Cesarean Section in a Pregnant Woman with COVID-19: First Case in Portugal. Acta Med Port. 2020;33(6): 429431. doi: 10.20344/amp.13883.

12. Yang $P$, Wang $X$, Liu $P$, Wei $C$, He B, Zheng J, et al. Clinical characteristics and risk assessment of newborns born to mothers with COVID-19. J Clin Virol. 2020;127: 104356. doi: 10.1016/j. jcv.2020.104356.

13. Zhang L, Jiang $Y$, Wei $M$, Cheng BH, Zhou XC, Li J, et al. Analysis of pregnancy outcomes of pregnant women during the epidemic of new coronavirus pneumonia in Hubei. Zhonghua Fu Chan Ke Za Zhi. 2020;55(3):166-171. doi: 10.3760/ cma.j.cn112141-20200218-00111.

14. Ministério da Saúde. Recomendações para o Trabalho de Parto, Parto e Puerpério Durante a Pandemia da Covid-19 Nota técnica no 9/2020 [internet]. Brasília: Secretaria de Atenção Primária à Saúde; 2020 [citado em: 25 jun 2020]. Disponivel em: http://docs.bvsalud. org/biblioref/2020/05/1095677/notatecnica92020cosmucgcividapessapsms.pdf.

15. Baergen RN, Heller DS. Placental Pathology in Covid-19 Positive Mothers: Preliminary Findings. Pediatr Dev Pathol. 2020;23(3): 177-180. Doi: 10.1177/1093526620925569.

16. Jie $P$, Xinghui $L$. Classification management recommendations for the full prevention and control of perinatal period under the outbreak of new coronavirus pneumonia. Chinese Journal of Obstetrics and Gynecology, 2020;55. doi: 10.3760/ cma.j.cn112141-20200221-00123.

17. Shanes ED, Mithal LB, Otero S, Azad HA, Miller ES, Goldstein JA. Placental Pathology in COVID-19. Am J Clin Pathol. 2020;154(1): 23-32. doi:10.1093/ajcp/aqaa089.

18. Chen S, Huang B, Luo DJ, LiX, Yang F, Zhao Y, et al. Clinical characteristics and placental pathology analysis of three pregnant women infected with novel coronavirus. J Perinatol. 2020;49(5):418-423. doi: 10.3760/cma.j.cn112151-20200225-00138.

19. Mimouni F, Lakshminrusimha S, Pearlman SA, Raju T, Gallagher PG, Mendlovic J. Perinatal aspects on the covid-19 pandemic: a practical resource for perinatal-neonatal specialists. J Perinatol. 2020: 40(5): 820-826. doi: 10.1038/s41372-020-0665-6.

20. Vivanti AJ, Vauloup-Fellous C, Prevot S, Zupan V, Suffee C, Cao JD, et al. Transplacental transmission of SARS-CoV-2 infection. Nature Communications. 2020;11(3572). doi: 10.1038/s41467-02017436-6.

21. Peng Z, Wang J, Mo Y, Duan W, Xiang G, Yi M, et al. Unlikely SARS-CoV-2 vertical transmission from mother to child: A case report. J Infect Public Health. 2020;13(5): 818-820. doi: 10.1016/j. jiph.2020.04.004.

22. Lang Guan-Jing, Zhao H. Can SARS-CoV-2-infected women breastfeed after viral clearance? J Zhejiang Univ Sci B. 2020; 21(5): 405-407. doi: 10.1631/jzus.B2000095.

23. Rasmussen SA, Smulian JC, Lednicky JA, Wen TS, Jamieson DJ. Coronavirus Disease 2019 (COVID-19) and pregnancy: what obstetricians need to know. Am J Obstet Gynecol. 2020; 222(5): 415-426. doi: 10.1016/j.ajog.2020.02.017.

24. Carvalho WB, Gibelli M ABC, Krebs VLJ, Calil VM Laurindo T, Johnston C. Expert recommendations for the care of newborns of mothers with COVID-19. Clinics. 2020; 75: e1932. doi: 10.6061/ clinics/2020/e1932.

25. Lowe B, Bopp B. COVI-19 vaginal delivery - A case report. Aust N Z J Obstet Gynaecol. 2020; 60(3): 465-466. doi: 10.1111/ajo.13173. 